across ethnicity. For example, CD4 T cells in African-Americans have a higher expression of type 1 and type 2 interferon pathways. In contrast, myeloid cells have several upregulated pathways in Caucasians, including ERK/MAPK signaling.

Conclusions African-American lupus nephritis patients may have a stronger interferon pathway activation in infiltrating immune cells. Several other pathways, including ERK/MAPK, are differentially expressed in infiltrating cells based on ethnicity. These results suggest that ethnicity might predict a response to both current and upcoming treatments, paving the way for a more personalized approach to treatment in lupus nephritis. Further work in Phase 2 of AMP will confirm and extend these findings.

Funding Source(s): NIH foundation partnership with AbbVie, Biogen, Bristol-Myers Squibb, Celgene, GlaxoSmithKline, Johnson and Johnson, Lilly, Merck, Pfizer, Sanofi, Takeda, and Verily.

\section{IMPACT OF THE BIRTH MONTH IN THE DEVELOPMENT OF SYSTEMIC LUPUS ERYTHEMATOSUS}

Renan Frittoli, Roberto Marini, Lilian Costallat, Simone Appenzeller*. University of Campinas

\subsection{6/lupus-2019-Ism.206}

Background There is evidence that most individuals with Systemic Lupus Erythematosus (SLE) have been born at the end of the winter season, mainly because of the influence of the mother's exposure to sunlight during pregnancy, possibly affecting vitamin D metabolism. The objective was to evaluate the influence of the birth month in the development of SLE.

Methods We included consecutive patients with childhoodonset SLE (cSLE) (age at onset of disease 16 years) and adultonset SLE (age of onset of disease $>16$ years) from the Rheumatology outpatient unit in Brazil. The control group consisted of volunteers no history of autoimmune disease. Through the review of medical records the patient's date of birth was obtained and the patients were classified according to the months and seasons of the year in which they were born. The results were presented in a descriptive way and the statistical analysis was performed through the chi-square test. For all analyzes $\mathrm{p}<0.05$ was considered statistically significant. Results A total of 1460 subjects (760 patients and 700 controls) were included. Of the patients analyzed, $662(87.1 \%)$ were adult-onset SLE and 98 cSLE (12.89\%). The mean age of the adult SLE was 42.4 years $(\mathrm{SD} \pm 12.7)$ and cSLE was 17.8 years $(\mathrm{SD} \pm 4.4)$. The controls had a mean age of 24.5 years (SD \pm 10.1 ). Patients who were born at the end of the winter season $[n=65(8.5 \%)]$ presented a statistically significant difference in relation to the control group $[n=55(7.8 \%)]$ $(p=0.011)$. When it was considered only patients with cSLE, it was observed a significantly higher birth numbers of cSLE patients during the winter season in Brazil (June 21-September 21) when compared to the controls $(p=0.018)$, and cSLE presented presented a birth frequency in winter (35.7\%) twice as high as those born in summer (17.34\%) and spring (17.34\%). A significant difference was also observed in cSLE in the month of August (which is winter in Brazil) $(p=0.042)$, when compared to the controls. Adult SLE had no differences with the control group in any month $(\mathrm{p}>0.05)$.

Conclusions It is believed that the winter season interferes with the development of SLE, especially in cSLE. These results may reinforce the idea that climate can be a contributing factor to the development of cSLE.

Funding Source(s): CNPq

FAPESP

\section{A HIGH GENETIC RISK SCORE IS ASSOCIATED WITH EARLY DISEASE ONSET, ORGAN DAMAGE AND DECREASED SURVIVAL IN SYSTEMIC LUPUS ERYTHEMATOSUS}

\begin{abstract}
${ }^{1}$ Sarah Reid*, ${ }^{2}$ Andrei Alexsson, ${ }^{3}$ Martina Frodlund, ${ }^{2}$ Johanna Sandling, ${ }^{2}$ Karin Bolin, ${ }^{4}$ Elisabet Svenungsson, ${ }^{5}$ Andreas Jönsen, ${ }^{6}$ Christine Bengtsson, ${ }^{7}$ Iva Gunnarsson, ${ }^{5}$ Anders A Bengtsson, ${ }^{8}$ Solbritt Rantapää-Dahlqvist, ${ }^{2}$ Maija-Leena Eloranta, ${ }^{2}$ Ann-Christine Syvänen, ${ }^{3}$ Christopher Sjöwall, 'Lars Ronnblom, ${ }^{1}$ Dag Leonard. ${ }^{1}$ Uppsala university; ${ }^{2}$ Dept of Medical Sciences, Science for Life Laboratories, Rheumatology, Uppsala University; ${ }^{3}$ Linköping University; ${ }^{4}$ Karolinska Insitutet; ${ }^{5}$ Dept of Rheumatology, Skåne University Hospital; ${ }^{6}$ Christine Bengtsson; ${ }^{7}$ Dept of Medicine, Karolinska Institutet; ${ }^{8}$ Dept of Public Health and Clinical Medicine/Rheumatology, Umeå University
\end{abstract}

10.1136/lupus-2019-Ism.207

Background Systemic lupus erythematosus (SLE) is a chronic, autoimmune disease with a complex genetic etiology. Over 100 risk genes for SLE have been identified at genome-wide significance, but their overall effect on disease severity has not previously been studied. We therefore assessed the relationship between a high genetic risk score and the development of organ damage in SLE.

Methods Patients with SLE, who met 4 ACR criteria $(\mathrm{n}=1001)$, and healthy controls $(\mathrm{n}=2802)$ were genotyped using a 200K Immunochip SNP Array (Illumina). A genetic risk score (GRS) was assigned to each individual based on 57 SLE risk loci which have previously shown association $(\mathrm{p}<5 \times 10-8)$ with SLE according to Chen et al (Curr Opin Rheumatol, 2017; 29(5):423-433), weighted by their SLE susceptibility odds ratios (ORs). Clinical data was retrieved from medical charts.

Results SLE prevalence increased with increasing GRS (figure 1A) and was higher in the highest compared to the lowest GRS-quartile (OR 12.32 (9.5315.71) $\quad \mathrm{p}=7.9 \times 10-86)$. SLE onset occurred 5 years earlier in the high compared to the low quartile (figure 1B). The OR for organ damage increased with increasing GRS (figure 1C) and was significantly higher in the high compared to the low GRS-quartile (OR 1.47 $(1.062 .04) \mathrm{p}=2.0 \times 10-2)$. Moreover, patients in the high quartile had an increased prevalence of nephritis (OR 2.22 (1.503.27), $\mathrm{p}=5.9 \times 10-5)$, end-stage kidney disease (ESKD) (OR 5.58 (1.5020.79), $\mathrm{p}=1.0 \times 10-2)$ anti-dsDNA antibodies (OR 1.83 (1.192.81), $\mathrm{p}=6.1 \times 10-03)$, anti-cardiolipin-IgG (OR 2.16 (1.303.59), $\mathrm{p}=2.8 \times 10-03)$ and anti-2-glycoprotein-I (OR 1.69 (1.042.74), $\mathrm{p}=3.3 \times 10-02)$. Analysis of renal biopsy data showed that the prevalence of proliferative nephritis was significantly higher in the high, compared to the low, quartile (OR 2.42 (1.304.49), $\mathrm{p}=5.1 \times 10-03)$. Moreover, the patients in the high GRS-quartile displayed decreased survival until their first organ damage (HR 1.51 (1.042.25), $\mathrm{p}=3.7 \times 10-02)$, first cardiovascular event (HR 1.65 (1.032.64), $\mathrm{p}=2.6 \times 10$ $02)$, nephritis onset (HR $2.53(1.723 .71) \mathrm{p}=9.6 \times 10-7)$ and ESKD (6.78 (1.7826.86), $\mathrm{p}=6.5 \times 10-3)$. Lastly, OR for mortality increased with increasing GRS (figure 1D), with a 5 year decrease in overall survival in the high compared to the low quartile (HR 1.82 (1.043.19), $\mathrm{p}=2.4 \times 10-2$ ).

Conclusions A high genetic risk score is associated with earlier disease onset, increased risk of organ damage and impaired 

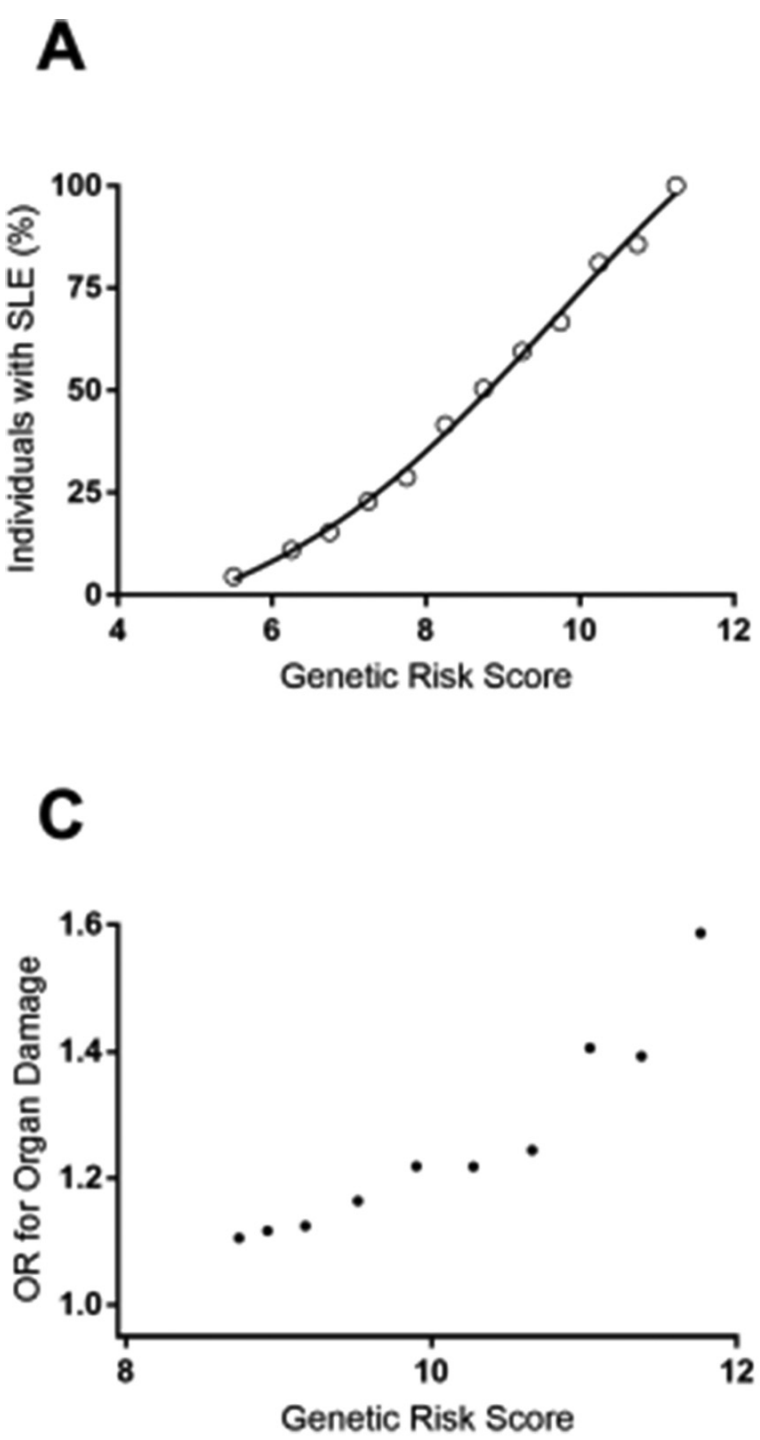

B

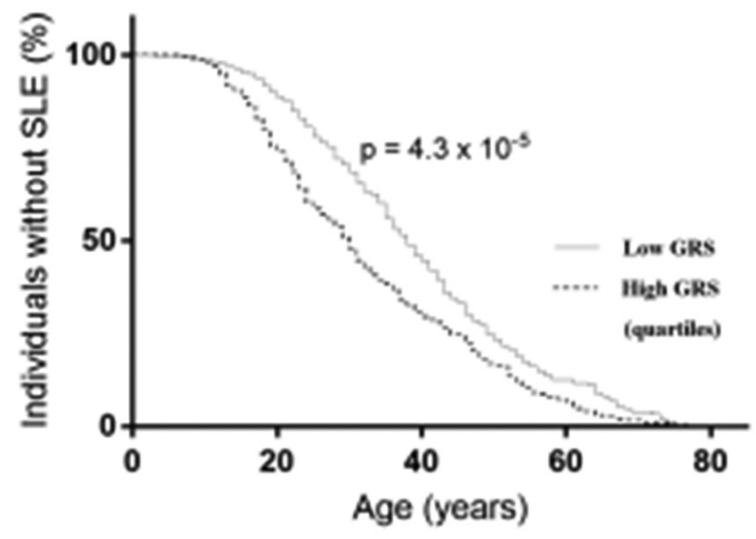

D

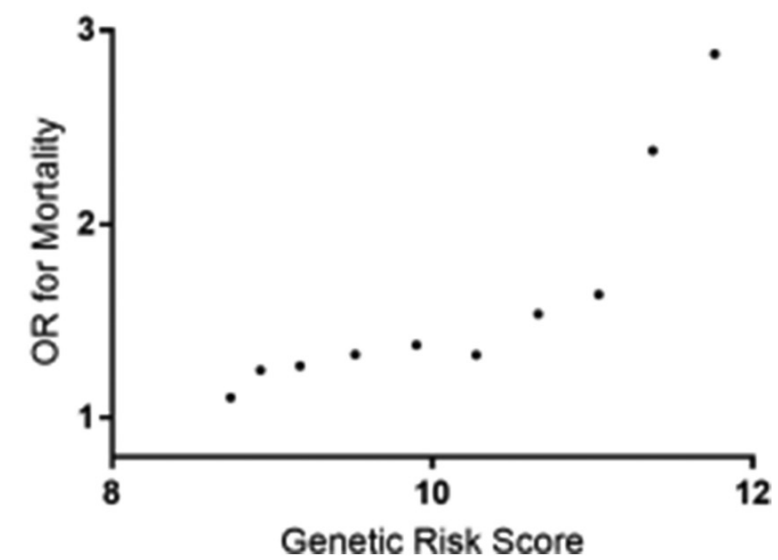

Abstract 207 Figure 1 Association of the genetic risk score with SLE onset, organ mortality. A) The prevalence of SLE in the patient-control population was plotted for groups with a GRS of $<6,6-6.5,6.5-7,7-7.5,7.5-8,8-8.5,8.5-9,9-9.5,9.5-10,10.5-11$ and $>11$. B) The survival until SLE onset was analysed for patients with a GRS in the extreme quartiles. $(n=500)$. C) IN regression analysis, the prevalence of organ damage in patients with a genetic risk score (GRS) above 7.0, 7.5, 8.0, 8.5, 9.0, 9.5, 10.0, 10.5, 11.0 and 11.5, respectively, was compared to a reference group with a GRS below 7.0. The odds ratio for each each group was plotted against the mean GRSs. Age was used as acovariate in each analysis D) Using the same method and groups as in C, odds ratio for mortality were plotted against mean GRSs.

survival. Our results indicate that genetic profiling may be useful for predicting outcomes in patients with SLE.

Funding Source(s): None

\section{8 (5R)-5-HYDROXYTRIPTOLIDE AMELIORATES NEPHRITIS IN LUPUS-PRONE MICE BY PREVENTING INFILTRATION OF IMMUNE CELLS}

${ }^{1}$ Jian-ping Zuo*, 'Shi-jun He, 'Ze-min Lin, ${ }^{2}$ Wei Tang. 'Laboratory of Immunopharmacology, State Key Laboratory of Drug Research, Shanghai Institute of Materia Medica, Chinese Academy of Sciences, Shanghai 201203, China; 'Laboratory of Immunopharmacology, Shanghai Institute of Materia Medica, Chinese Academy of Sciences, Shanghai 201203, China

10.1136/lupus-2019-|sm.208

Background (5R)-5-hydroxytriptolide (LLDT-8), a triptolide derivative with low toxicity, was previously reported to have strong immunosuppressive effects both in vitro and in vivo, but it remains unknown whether LLDT-8 has a therapy effect on systemic lupus erythematosus (SLE).

Methods In vivo, the therapeutic effects of LLDT-8 in MRL/ lpr mice were investigated. Ex vivo, the mechanisms of treatment were explored according to the immunologic correlates of disease. In vitro, human proximal tubule epithelial cell line and mouse mesangial cell line were used to evaluate the regulatory effects of LLDT-8 on chemokine expression.

Results Compared with the vehicle group, different clinical parameters were improved upon LLDT-8 treatment as follows: prolonged life span of mice, decreased proteinuria, downregulated blood urea nitrogen and serum creatinine, reduced glomerular IgG deposits, and ameliorated histopathology. A decreased expression of the inflammatory cytokines IFN- $\gamma$, IL17, IL- 6 , and TNF- $\alpha$ was also observed in the kidney of LLDT-8 treated MRL/lpr mice. Moreover, infiltration of $\mathrm{T}$ cells in the kidney was mitigated after LLDT-8 treatment, 